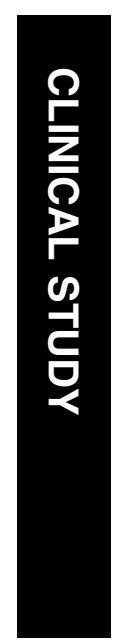

\title{
Glaucoma electronic patient record-design, experience and study of high-risk patients
}

${ }^{1}$ Department of Ophthalmology, Ninewells Hospital, Dundee, UK

${ }^{2}$ Department of Ophthalmology, Queen Margaret Hospital, Dunfermline, UK

Correspondence: R Sanders, Department of Ophthalmology, Queen Margaret Hospital, Whitefield Road, Dunfermline, Fife KY12 OSU, UK

Tel: + 1383 623623;

Fax: + 1383674009

E-mail: roshini.sanders@ faht.scot.nhs.uk

Received: 19 November 2004

Accepted in revised form:

6 June 2005

Published online:

29 July 2005

Presentations: Royal College of Ophthalmologists Annual Congress, May 2003 HCMMI Awards for the best use of IT in a clinical sector, London 2002.

Competing interests: none declared

\begin{abstract}
Aim To set-up a glaucoma electronic patient record (GEPR) and study referral details to a new glaucoma service, concentrating on highrisk patients.

Method A GEPR was designed using the pre-existing hospital information technology (IT) infrastructure. Referral details of all new patients to the glaucoma service were completely electronically entered and analysed.

Results A GEPR was successfully established. A total of 402 referrals were studied. In all, $43 \%$ (40) of high-risk clinic patients (IOP $>29 \mathrm{mmHg}$, or C/D ratio $>0.8$ or moderate to advanced visual field defects) had to wait longer than 8 weeks from GP referral to be seen at the glaucoma service. Of these, nine patients lost more than one line of Snellen's visual acuity attributable to glaucoma. The optometrist failed to document IOP in $17 \%$, fundoscopy in $30 \%$, and visual fields in $45 \%$ of all referrals.

Conclusions A GEPR can be introduced in an NHS setting without disruption of clinical care and ophthalmic training, and facilitates detailed, accurate and rapid audit. Study of high-risk glaucoma referrals showed inadequate optometric referral details and poor prioritisation of urgent cases. This information is being utilised with the cooperation of local optometrists to refine the pattern of glaucoma referrals.

Eye (2005) 19, 956-962. doi:10.1038/sj.eye.6702008; published online 29 July 2005
\end{abstract}

Keywords: electronic record; high-risk glaucoma; optometry
F Imrie1, A Blaikie1, C Cobb², A Sinclair², D Wilson², $S$ Dobson ${ }^{2}$ and $R$ Sanders ${ }^{2}$

\section{Introduction}

The government white paper 'Information for Health' has mandated the use of level 3 EPRs in all acute hospitals by $2005 .^{1}$ The Queen Margaret is a new 949 bedded hospital where a uniform information technology (IT) system Oasis was installed throughout the hospital to support both administrative and clinical needs. Oasis is an open and integrated clinical IT system that can operate beyond EPR level 6. ${ }^{2}$ The hospital is well equipped with computers, has a 14-man IT team, an on call IT officer, a lead physician and close links with Capula Limited, the providers of Oasis. This supportive IT environment enabled us to develop a Glaucoma electronic patient record (GEPR) in 2000.

A new consultant led glaucoma service was set-up in 1998 seeing all new referrals with possible glaucoma in addition to providing the regional glaucoma training needs of SpRs. The waiting time for new patients gradually rose from 6 to 24 weeks and within 1 year of the service new referral numbers doubled. Prioritisation of urgent referrals thus became an immediate need. Fraser $e t a l^{3}$ have estimated that an incorrect optometric glaucoma referral is over four times more likely to be a late presenter than a comparable patient with appropriate referral. Tuck and Crick $^{4}$ have shown that $10 \%$ of high-risk glaucoma patients had to wait at least 14 weeks for an NHS appointment.

This paper describes our experience of design, installation, use, and benefits of a GEPR. As the first of a series of reports made possible by the GEPR we chose to analyse referral details of all patients, concentrating on high-risk patients. 


\section{Methods}

\section{Glaucoma electronic patient record}

The GEPR was designed within Oasis in 2000 by DW, RS, $\mathrm{AB}$, and Capula Limited (a major national and international provider of innovative IT health services) using the Oasis clinical tool sets, a generic system that enabled clinicians to design their own systems using recognised open technologies, Java, and XMEL. The only precedent in the UK at that time for an ophthalmic EPR was the vitreoretinal EPR at Moorfileds Eye Hospital. ${ }^{5}$

The GEPR was designed to incorporate all information previously recorded in the handwritten glaucoma clinical records but with greater detail and additional optometric referral details. Altogether, the GEPR had 42 stem questions, which defaulted to a range of common preset answers. Rare conditions or findings required free text. The design was such that each section required completion in order to default to the next section. Users were required to complete the GEPR in a standardised manner not possible with the written record.
The GEPR generated automatic GP and optician discharge summaries and patient information letters. Although Oasis had its own drawing package developed in Java applets we found this to be as inaccurate as the free drawn images in clinical notes. Therefore, all new patients had stereodisc photographs inserted in their case notes and images stored on our digital photography system accessible using a standard integration package (Oasis interface engine). In addition, the GEPR had detailed accounts of disc parameters that required completion (Figure 1). Anterior segment abnormalities were also photographed and stored in a fashion similar to disc photographs. Special software was required to link Oasis to the Humphrey visual field analyser and this development is currently being investigated. The IT specifications utilised for the GEPR are outlined in Table 1. All patient information was entered at the glaucoma clinic by RS, AS, FI, and AB, each of whom had a unique username. The GEPR had multiple access and required signing off after completion. Once signed off the GEPR could not be electronically amended but was

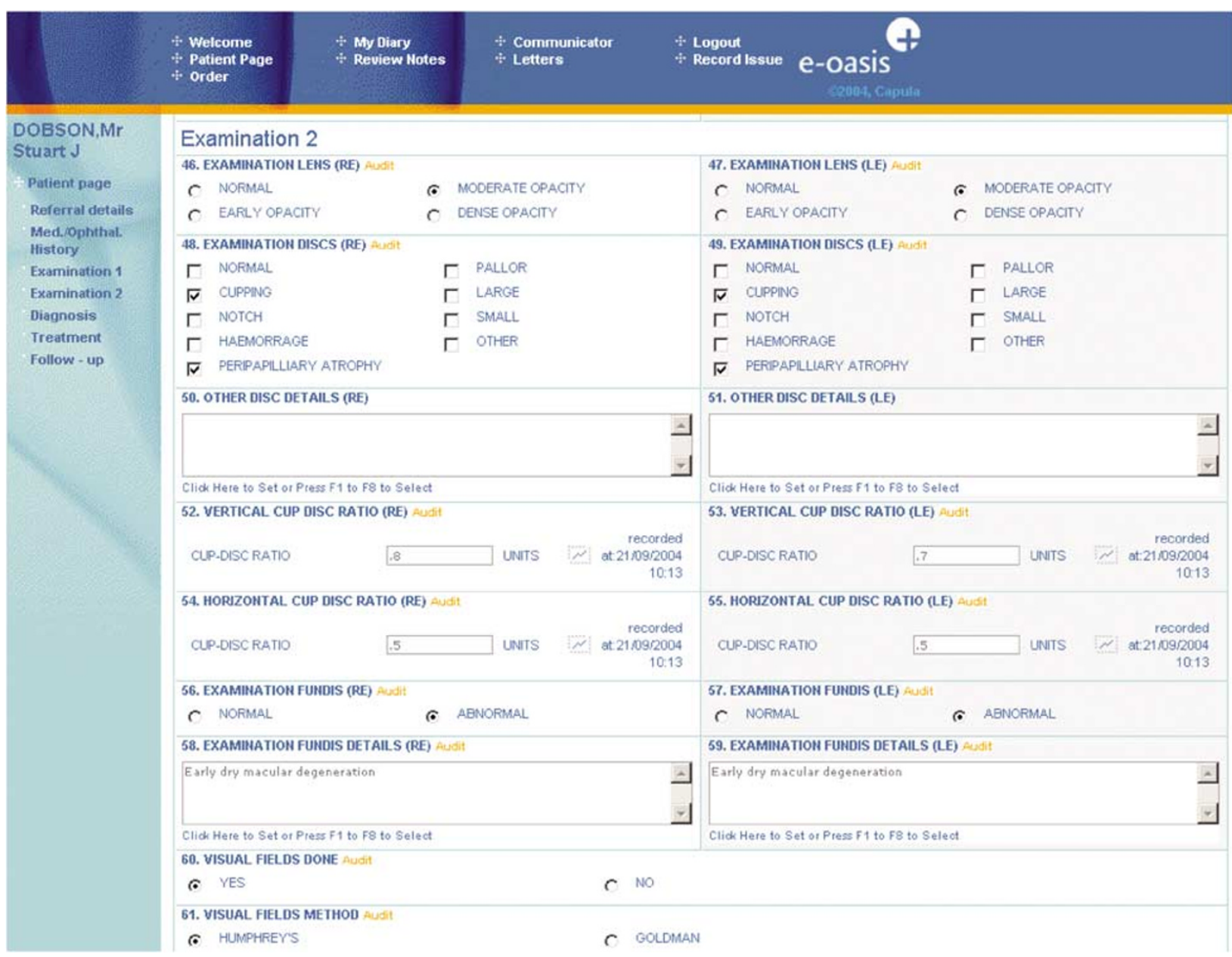

Figure 1 Still of GEPR with details of ophthalmic examination. 
Table 1 The IT details of computer soft- and hardware and network requirements for the GEPR

Glaucoma clinic computers-Dell Optiplex GX270, P4 2.00 Ghz, $512 \mathrm{Mb}$ RAM

Operating systems - Windows 2000/XP

Database software - Oracle

Intranet browser-Microsoft Internet Explorer Version 5.5

Network - 10 Base T Ethernet

Network software-Windows 2000/Citrex Metaframe XP

available as a shared record for viewing by other hospital staff. There was also an audit trail in place that recorded details of all GEPRs accessed within the hospital and so acted as security for patient information required under the Data Protection Act 1998 and Coldicott Guidelines. ${ }^{6}$ Hospital policy dictated that a printed paper copy of the GEPR was placed in the clinical notes for ease of access by other clinicians throughout the hospital.

A few training sessions were required before live use of the system in 2000. The clinic room layouts required alteration. Where previously equipment was laid out randomly, a more focused arrangement of slitlamp, computer, and printer, all side by side, was made in an attempt not to detract from the patient-doctor consultation relationship and eye contact.

A time in motion study at the initial stages revealed an average time of $15 \mathrm{~min}$ for individual patient data entry. The repercussions were an unacceptable waiting time for patients and lengthy clinics. The SpR training was compromised due to pressure on clinic time. During the first 6 months the GEPR was constantly modified by the users, resulting in more short cuts and defaults. All redesign was incorporated by DW. Capula either e-mailed or were on site to make alterations to the Oasis clinical tool sets that were beyond the scope of DW. A repeat time in motion study 6 months later showed that the average time for data entry was reduced to $9 \mathrm{~min}$. This was the time taken for new patient data entry, which included detailed entry of optometric referral details , that were not recorded in previous handwritten clinical notes. In addition, all new patients had fundoscopy following dilation of pupils and time was lost electronically opening and closing individual files twice. Since then short cuts have been developed within the software and data entry time has been further shortened. Follow-up patients took less than five minutes for electronic data entry. Any difficulties with use of the GEPR at the clinic were immediately addressed by an on call IT officer.

Data from the GEPR was automatically transferred to the hospital data warehouse in Oracle. Analytical software in the form of Business Objects (a powerful IT reporting tool $^{7}$ ) was used by SD to retrieve primary and linked data from the data warehouse. In addition, the GEPR was exported to Excel, which was found to be more user friendly for audit presentation purposes.

All information on high-risk clinic patients (92) and those discharged at their first clinic visit (96) was manually verified and no error was found apart from one patient who had another patient's GEPR filed in the notes. This being the case, the information retrieved from the remaining 214 patients was assumed to be accurate.

\section{Glaucoma audit}

The GEPR had a detailed account of optometric referral details unlike the previous written clinical notes (Figure 2). This included the date of optometric and GP referral dates and optometric clinical data that included the highest optometric intraocular pressure (IOP) reading, fundoscopy, and visual field findings. An optometric disc finding of a cup/disc $(C / D)$ ratio $>0.4$, an asymmetry of $C / D$ ratio $>0.1$, as well as other disc abnormalities such as haemorrhage, notching, pallor or size were all documented as an abnormal optometric disc finding. These parameters were chosen as a $C / D$ ratio $>0.4$ is known to occur in less than $10 \%$ of the normal population $^{8}$ and an asymmetry of $C / D$ ratio $>0.1$ in less than $7 \%$ of the population. ${ }^{9}$ Any visual field abnormality picked up by optometrists was documented as an abnormal optometric visual field finding.

All referrals to the glaucoma service were prioritised by RS based on optometry referral details. High-risk referrals were classified as those patients who had an optometric finding in one or both eyes of IOP $>29 \mathrm{mmHg}$ or a $C / D$ ratio $>0.8$ or a moderate or advanced visual field defect. The latter was defined as a field defect within $5-10^{\circ}$ of fixation. ${ }^{10}$ An IOP $>29 \mathrm{mmHg}$ was chosen as previous work has shown that an optometric IOP $>29 \mathrm{mmHg}$ resulted in a diagnosis of glaucoma being made in $65 \%$ of patients. ${ }^{4}$ A $C / D$ ratio $>0.8$ was chosen as the range of a normal $C / D$ ratio is known to lie between 0 and $0.8 .{ }^{11}$ Any one or more of the above referral details resulted in an appointment being made for the glaucoma service within 4 weeks.

All new patients had a full examination by an experienced ophthalmologist (RS, AS, FI, or AB). A disc was deemed abnormal if the $C / D$ ratio was $>0.4{ }^{8}$ if it had an asymmetry of $C / D$ ratio $>0.1^{9}$ or other abnormalities such as pallor, haemorrhage, notching, or abnormalities of size.

A high-risk clinic glaucoma patient was classified as any patient who at their first clinic visit had in one or both eyes an IOP $>29 \mathrm{mmHg}$ as measured with Goldmann tonometry or a $C / D$ ratio $>0.8$ or a moderate or advanced visual field defect. ${ }^{10}$ All visual fields were 


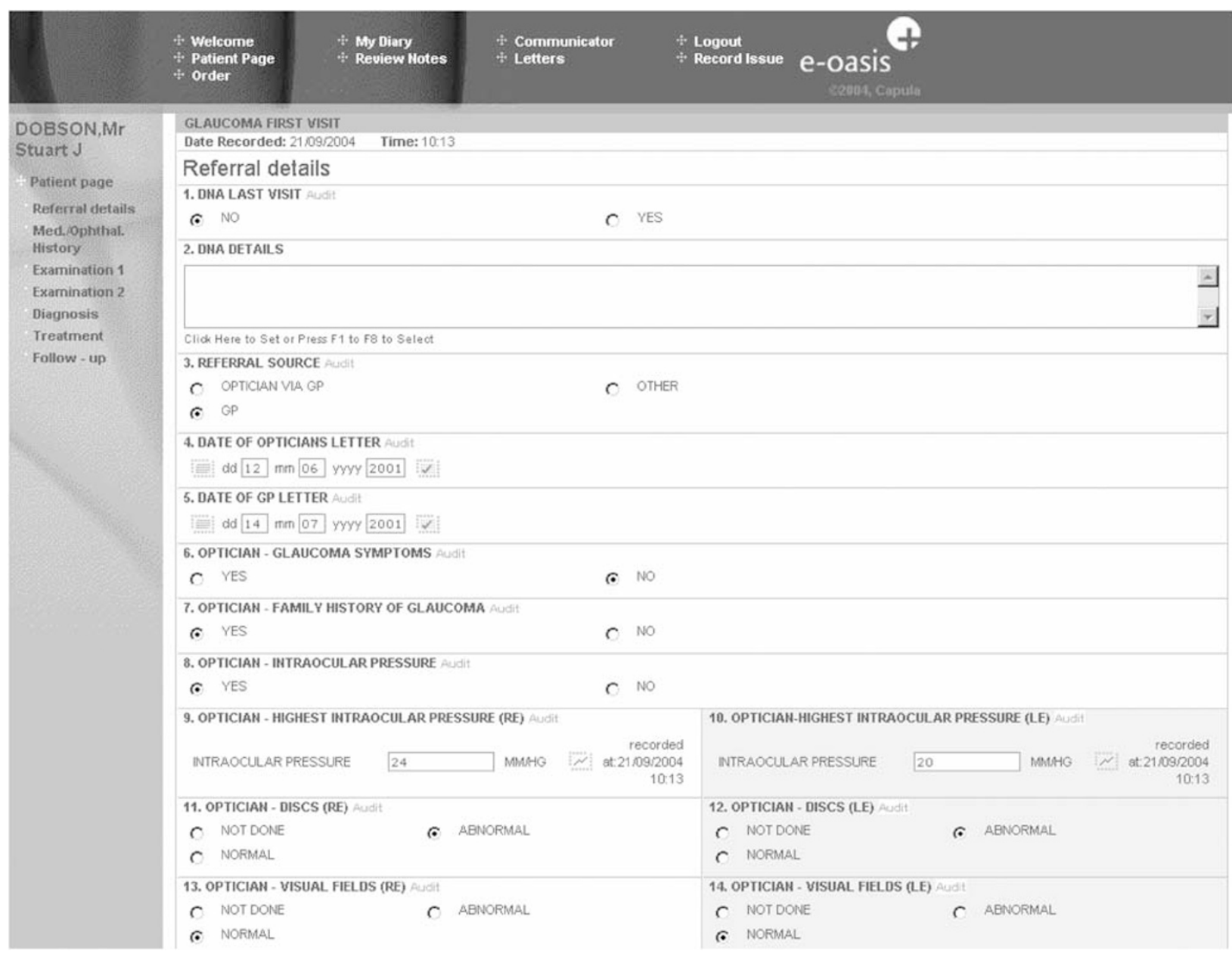

Figure 2 Still of GEPR with details of optometric referral.

examined and reported by one reporter for consistency (RS). All patients had a Humphrey Sita Standard 24-2 degree threshold visual field test. In a few circumstances when this was not possible due to patient factors a Goldmann field was charted. In cases of poor fixation, perimetry was repeated to obtain the best possible result. A glaucomatous field defect was documented after obtaining the best possible visual field and excluding other causes of field deficit such as macular degeneration or vascular occlusion.

\section{Results}

\section{Glaucoma EPR}

By 2002, 402 new referrals had been successfully entered into the GEPR which allowed us to conduct our first audit. The glaucoma clinics ran to time with no compromise of patient care or SpR training. The patients have contributed positive comments and feel they are attending a state of the art computerised glaucoma clinic. There have been no complaints regarding compromise of clinical care and some have requested an electronic summary of their glaucoma care. We continue to this day with designing upgrades and modifications to the GEPR, which is being used by all who pass through the glaucoma service, including new staff and glaucoma specialist nurses. We have been encouraged by the speed and accuracy with which detailed clinical information can be retrieved. Graph 1 shows the optometrist and clinic IOP documentation for all new referrals to the glaucoma service. This graph was produced in 10 minutes and is an example of rapid access and accurate presentation of clinical data.

\section{Glaucoma audit}

Of the 402 new patients entered into the GEPR, 20 were tertiary or interhospital referrals. This study confines 

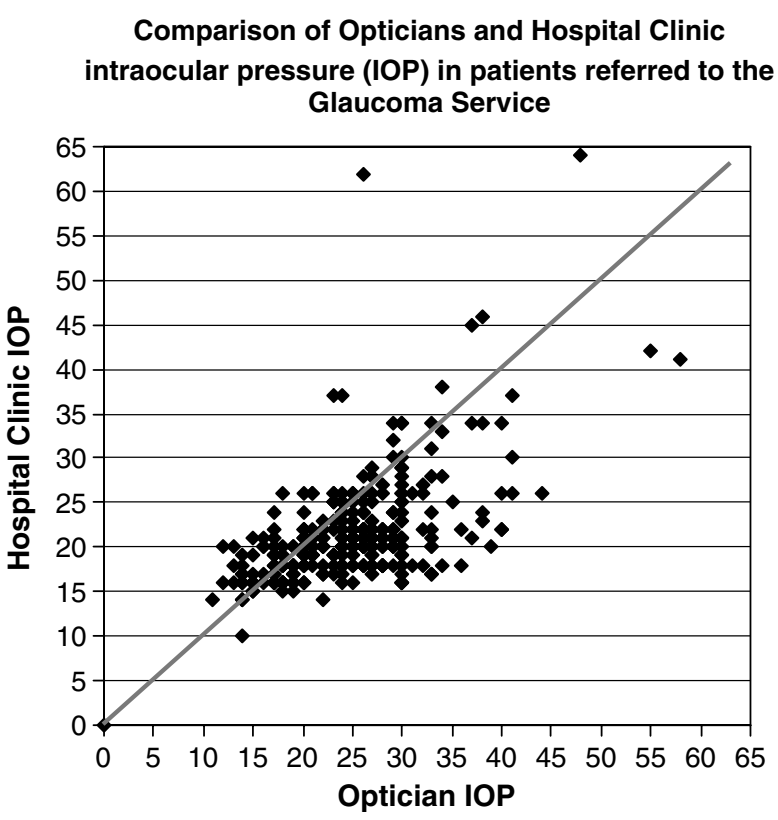

Graph 1 Direct comparison of optometry and hospital intraocular pressure (IOP) readings for all new patients referred to the glaucoma service.

itself to details regarding referrals of the remaining 382 patients by local optometrists.

For the whole group (382 patients), the difference in weeks between optometric and GP referral were within 1 week in $172(45 \%), 2$ weeks in $107(28 \%), 3$ weeks in $31(8 \%)$, and 3-17 weeks in $46(12 \%)$. In $26(7 \%)$ patients, the date of optometric referral was absent.

The difference in weeks between GP referral and first hospital appointment was within 4 weeks in $38(10 \%)$,

8 weeks in $138(36 \%), 12$ weeks in $88(23 \%)$, and in excess of 12 weeks in $88(23 \%)$. In all, $30(8 \%)$ patients failed to attend their first glaucoma clinic appointment and required recall.

Of the 382 referrals, 96 (25\%) patients were discharged on their first clinic visit after normal IOP findings, funduscopy, and visual field testing. A further 96 (25\%) were discharged after a second or third visual field test. Eighty four (22\%) were followed up as glaucoma suspects, and $106(28 \%)$ had a positive diagnosis of glaucomatous disease or ocular hypertension.

For the whole group of 382 patients the optometrist checked IOP using Goldmann's or puff tonometry in 317 (83\%) and performed fundoscopy in 267 (70\%).

Optometric perimetry was performed in $212(55 \%)$ with a Henson's analyser in 68 (32\%), Humphrey in 43 (20\%) and Friedman, Dicon, and FDT in 40 (19\%). In 61 (29\%) cases the visual fields were not enclosed with the referral and the strategy used was not mentioned. Less than 21 $(10 \%)$ had repeat perimetry.
In all, 32 patients had a clinic IOP $>29 \mathrm{mmHg}$ in one or both eyes. Of these patients only $16(50 \%)$ were seen within 8 weeks of GP referral. Of the other 16, four patients suffered a loss of Snellen's visual acuity of more than one line from GP referral to hospital visit, attributable to glaucoma. In these 32 patients, 17 had an optometric IOP $>29$, nine an IOP $<30$, and six had no documentation of IOP.

In all, 80 patients had a clinic cup disc $C / D$ ratio of $>0.8$ in the vertical or horizontal meridian in one or both eyes. Of these 27 (34\%) were seen within 8 weeks of GP referral. Of the other 53, four suffered a loss of Snellen's visual acuity of more than one line, between GP referral and hospital visit, attributable to glaucoma. Of these 80 patients, 40 also had an abnormal optometry disc finding, nine a normal optometry disc finding, and 31 had no documentation of fundoscopy. A direct comparison of normal and abnormal optometric and clinic optic disc appearances for the whole group gave a $10.4 \%$ (95\% CI, 5.3\%, 15.6\%) false negative and $36.1 \%$ (95\% CI, $27.9 \%, 44.3 \%)$ false-positive rate.

In all, 73 patients had a moderate or severe visual field loss due to glaucoma in one or both eyes at the clinic as documented by Humphrey or Glodmann perimetry. Of these, $24(33 \%)$ were seen within 8 weeks of GP referral. Of the other 49 , six suffered a loss of Snellen's visual acuity of more than one line, between GP referral and hospital visit, attributable to glaucoma. Of these 73 patients the optometrist found an abnormal field in 33 patients, a normal field in six patients, and did not perform perimetry in 34 patients. A direct comparison of optometric and clinic visual fields for the whole group gave an $11.1 \%$ (95\% CI, 3.9\%, 18.4\%) false negative and $46.4 \%$ (95\% CI, 38.2\%, $54.7 \%$ ) false-positive rate.

A total of nine patients lost more than one line of Snellen's visual acuity attributable to glaucoma between GP referral and hospital visit, five of whom had two separate high-risk clinic signs. The clinical notes of these nine patients were manually, carefully examined again taking into account patient history and comparison of optometric and clinical findings. All had optometric refraction and their best possible visual acuity documented at the clinic with correction, unaided, and with pin hole. All had suffered uniocular visual loss secondary to advanced glaucomatous disease with no compounding disease such as macular degeneration or vascular occlusion.

A total of 92 patients had one or more high-risk clinic signs of which $52(57 \%)$ were seen within 8 weeks of GP referral. In all, 29 patients had an abnormal IOP, disc, and visual field finding by the optometrist. Of this group glaucomatous disease was diagnosed in 24 (83\%) and 27 (93\%) were seen within 8 weeks of GP referral. 


\section{Discussion}

\section{Glaucoma EPR}

We have shown that it is possible to successfully establish a GEPR in an NHS setting. We know this to be the second longest running ophthalmic EPR in the UK. We feel this is a substantial achievement given that at the time of inception (2000) there was no other ophthalmic precedent apart from the viteoretinal EPR at Moorfields Eye Hospital. Presently there are other examples of GEPRs in the UK, based on systems that had to be financed and introduced into the hospital IT structure. Our GEPR is unique in that it was developed using the preexisting hospital IT system and so incurred no extra cost and had the support of the Hospital IT staff. This we feel is crucial to the success of any EPR and points to an alternative method of developing an EPR as opposed to importing new and expensive systems.

The uniform method of documenting patient details has advantages in continuing care for the patient and provides a sound training basis for the SpRs. An added benefit is that the GEPR provides ready, rapid retrieval and subsequent analysis of detailed patient information. This has empowered us with the information needed to negotiate with the hospital trust, optometrists, GPs, and health authorities for improvements and added resource for the glaucoma service. Presentation of clinical information and audit plays an increasing role in negotiating for resources within the competitive market of the NHS and the EPR has the potential to provide this.

\section{Glaucoma audit}

This study concentrated on referral patterns from optometrists and time taken to see high-risk clinic glaucoma patients. We found unnecessary delay between optometric and GP referral. Inadequate optometric referral data led to inefficient prioritisation of urgent cases. We realise that we have assumed the clinic findings to be the gold standard compared to the optometric findings. In our defence we should say that all patients were examined by an experienced ophthalmologist with a uniform method of investigation, instrumentation, follow-up, and repeat testing.

The failure of optometrists to perform all three glaucoma-screening tests (IOP, fundoscopy, and perimetry) in a consistent way, the variable equipment for testing as well as the failure to retest meant that the standard of referral was varied leading to an overall false-positive referral rate of $50 \%$.

Of grave concern are the nine patients who lost more than one line of Snellen's visual acuity resulting from untreated glaucoma while awaiting an NHS appointment. We chose a loss of more than one line of Snellen's visual acuity as we realise that there could simply be discrepancies of one line between different testers. Careful study of the individual cases concerned left us in no doubt that visual loss was secondary to untreated glaucoma. It is beyond the scope of this paper to describe each case in detail. However, significant factors we feel that contributed to the delay in hospital treatment were failure of the optometrist to perform all three glaucoma screening tests in six cases leading to poor prioritisation of hospital appointment, unnecessary delay between the optometry referral and GP referral to hospital in three cases, failure of the patient to attend the first hospital appointment in two cases, and inappropriate initial referral to a cataract clinic in one case.

At a local level, we have shared these results with our optometrists, trust management, and electronic implementation group. This has resulted in optometric training sessions, standardised minimum information with electronic referral, and re-examination of patients all at different stages of development.

At a national level it is of concern that there still remains wide disparity in optometric referral patterns ${ }^{12,13}$ and waiting times in the NHS for Glaucoma patients. ${ }^{14}$ Glaucoma suspects constitute the second commonest referral to the HES. ${ }^{13}$ The large numbers and variable referral information lend itself to poor prioritisation with the result that those with irrevocably blinding disease are not seen in time. Other studies have had variable success in attempting to refine their glaucoma referral patterns. ${ }^{15,13}$ We have the advantage of a stable patient population, good local communication, and cooperation with 34 optometry practices and strong hospital IT links, all of which will hopefully make a difference when we electronically reaudit our referral patterns in 2006.

\section{Acknowledgements}

We are grateful to M Mellor, CapulaTechnologies, London. J Wardrope (IT), P Curry, Consultant Anaesthetist, Queen Margaret Hospital, Dunfermline.

\section{References}

1 NHS Executive. Information for Health. London: DoH, 1998.

2 Website for Oasis - http://www.capula.co.uk/caphealthproducts.htm

3 Fraser S, Bunce C, Wormald R. Retrospective analysis of risk factors for late presentation of chronic glaucoma. $\mathrm{Br} J$ Ophthalmol 1999; 83: 24-28.

4 Tuck MW, Crick RP. Efficiency of referral for suspected glaucoma. BMJ 1991; 302: 998-1000. 
5 Aylward GW, Parmar DN. Information technology in ophthalmology - experience with an electronic patient record. BJO 1999; 83: 1264-1267.

6 Report on the Review of Patient Identifiable Information (1997). The Coldicott Committee. Department of Health: London.

7 Business Objects Foundation. Caci Information Solutions, Version 1, May 2002

8 Armaly MF. The optic cup in the normal eye. Cup width, depth, vessel displacement, ocular tension and outflow facility. Am J Ophthalmol 1969; 68: 401.

9 Leibowitz HM, Krueger DE, Maunder LR, Milton RC, Kini MM, Kahn HA et al. The Framingham eye study monograph. Surv Ophthalmol 1980; 24(Suppl): 335.
10 Murdoch J, Jay J. The rate of visual field loss in untreated primary open angle glaucoma. Br J Ophthalmol 1994; 77: 176-178.

11 Jonas JB, Naumann GOH, Werner EB. Normal cup-to-disc ratios (correspondence). Ophthalmology 1991; 98: 273.

12 Tuck M, Crick R. Glaucoma screening by optometrists: ten years on. Health Trends 1998/1999; 30(4): 130-134.

13 Vernon SA, Ghosh S. Do locally agreed guidelines for optometrists concerning the referral of glaucoma suspects influence referral practice? Eye 2001; 15: 458-463.

14 Tuck M, Crick RP. How can we improve the detection of glaucoma? BMJ 1995; 310: 546-547.

15 Henson DB, Spencer AF, Harper R, Cadman EJ. Community refinement of glaucoma referrals. Eye 2003; 17: 21-26. 